\title{
Bondi Accretion and the Problem of the Missing Isolated Neutron Stars
}

\section{Citation}

Perna, Rosalba, Ramesh Narayan, George Rybicki, Luigi Stella, and Aldo Treves. 2003. “Bondi Accretion and the Problem of the Missing Isolated Neutron Stars." The Astrophysical Journal 594 (2): 936-42. https://doi.org/10.1086/377091.

\section{Permanent link}

http://nrs.harvard.edu/urn-3:HUL.InstRepos:41384948

\section{Terms of Use}

This article was downloaded from Harvard University's DASH repository, and is made available under the terms and conditions applicable to Other Posted Material, as set forth at http:// nrs.harvard.edu/urn-3:HUL.InstRepos:dash.current.terms-of-use\#LAA

\section{Share Your Story}

The Harvard community has made this article openly available.

Please share how this access benefits you. Submit a story.

Accessibility 


\title{
Bondi Accretion and the Problem of the Missing Isolated Neutron Stars
}

\author{
Rosalba Perna ${ }^{1,2}$, Ramesh Narayan ${ }^{2}$, George Rybicki², Luigi Stella ${ }^{3}$ and Aldo Treves ${ }^{4}$
}

\begin{abstract}
A large number of neutron stars $(\mathrm{NSs}), \sim 10^{9}$, populate the Galaxy, but only a tiny fraction of them is observable during the short radio pulsar lifetime. The majority of these isolated NSs, too cold to be detectable by their own thermal emission, should be visible in X-rays as a result of accretion from the interstellar medium. The ROSAT all sky survey has however shown that such accreting isolated NSs are very elusive: only a few tentative candidates have been identified, contrary to theoretical predictions that up to several thousands should be seen. We suggest that the fundamental reason for this discrepancy lies in the use of the standard Bondi formula to estimate the accretion rates. We compute the expected source counts using updated estimates of the pulsar velocity distribution, realistic hydrogen atmosphere spectra, and a modified expression for the Bondi accretion rate as suggested by recent MHD simulations, and supported by direct observations in the case of accretion around supermassive black holes in nearby galaxies and in our own. We find that, whereas the inclusion of atmospheric spectra partly compensates for the reduction in the counts due to the higher mean velocities of the new distribution, the modified Bondi formula dramatically suppresses the source counts. The new predictions are consistent with a null detection at the ROSAT sensitivity.
\end{abstract}

Subject headings: accretion, accretion disks - X-rays: ISM - X-rays: stars

\footnotetext{
${ }^{1}$ Harvard Society of Fellows, 74 Mount Auburn Street, Cambridge, MA 02138

${ }^{2}$ Harvard-Smithsonian Center for Astrophysics, 60 Garden Street, Cambridge, MA 02138

${ }^{3}$ Osservatorio Astronomico di Roma, Sede di Monteporzio Catone, Via Frascati 33, I-00040 Rome, Italy

${ }^{4}$ Dipartimento di Scienze, Universita' dell'Insubria, Via Valleggio 11, I-22100 Como, Italy
} 


\section{Introduction}

Neutron stars (NSs) are a common endpoint of stellar evolution. Nucleosynthesis constraints on Galactic chemical evolution require that the total number of NSs in our Galaxy today be on the order of $10^{9}$ (Arnett, Schramm \& Truran 1989). After they are born, NSs are visible for a short while either as X-ray sources from their cooling radiation or as radio sources from pulsar activity. These phases, however, only last $\sim 10^{6}$ and $\sim 10^{7}$ $\mathrm{yr}$, respectively, which is a small fraction of the age $\sim 10^{10} \mathrm{yr}$ of the Galaxy. After a few tens of millions of years both the thermal and the rotational energy supply will be exhausted, and from then on the NSs would just be cold, invisible objects, if it were not for accretion from the interstellar medium (ISM), which makes them shine again in the X rays, as it was realized some 30 years ago by Ostriker, Rees \& Silk (1970). The problem received close attention in the early 1990s. According to the calculations of Treves \& Colpi (1991) and Blaes \& Madau (1993; BM in the following), the luminosity due to accretion can be significant, and as many as 10,000 NSs should be detectable with the ROSAT all sky survey.

The problem of the "Missing Neutron Stars" became evident when the observations returned only a handful of possible candidates. Subsequent revisions of the expected rates have somewhat alleviated the problem by accounting for other effects not included in the BM calculations, such as dynamical heating (Madau \& Blaes 1994), coupling of the dynamical and magnetorotational evolution (Popov et al. 2000; Livio, Xu \& Frank 1998; Colpi et al. 1998). Furthermore, in the last few years, more accurate estimates of pulsar velocities (Lorimer, Bailes \& Harrison 1997; Hansen \& Phinney 1997; Cordes \& Chernoff 1998; Arzoumanian et al. 2002) seemed to point towards an increase in the mean velocity (and hence a decrease in the accretion rate) relative to the Narayan \& Ostriker (1990) distribution used by BM. Whereas larger typical velocities imply smaller accretion luminosities, on the other hand, as we show here (see also Zane et al. 1995), the inclusion of more realistic spectra which go beyond the simple blackbody spectrum, tends to increase the predicted X-ray number counts ${ }^{5}$ and hence partly counterbalance the reduction due to other effects.

In this paper, we propose that a likely solution to the missing neutron stars problem lies in the use of the Bondi expression to calculate the accretion rate. Since the work of Fabian \& Canizares (1988), it has been known that the accretion luminosities of supermassive black holes in most galactic nuclei are much too low to be consistent with radiatively efficient accretion at the Bondi rate from the surrounding ISM. The evidence has become

\footnotetext{
${ }^{5}$ This is due to the fact that, for the same total luminosity, the spectrum from a light element atmosphere is significantly harder than a blackbody (see $\S 2.3$ for more details).
} 
compelling in recent years (Di Matteo et al. 2000, 2001; Loewenstein et al. 2001; Narayan 2002). Two solutions to this problem have been proposed (see e.g. Narayan 2002 for a review): (i) Radiatively inefficient accretion, as in an advection-dominated accretion flow (Ichimaru 1977; Rees et al. 1982; Narayan, Yi \& Mahadevan 1995; Fabian \& Rees 1995); (ii) Accretion at below the Bondi mass accretion rate (Blandford \& Begelman 1999; Narayan, Igumenshchev \& Abramowicz 2000; Quataert \& Gruzinov 2000). The current consensus is that both effects operate, with radiatively inefficient accretion triggering a reduction in the mass accretion rate $\dot{M}$. The case for a reduced $\dot{M}$ has become especially strong with the recent detection of linear polarization at $230 \mathrm{GHz}$ from $\mathrm{Sgr} \mathrm{A}^{*}$ at the Galactic Center (Bower et al. 2003). This observation indicates that the gas density close to the black hole is much less than that predicted for Bondi accretion.

In the context of the missing neutron star problem, we note that radiatively inefficient accretion alone will not solve the problem since the advected energy will be radiated once the accreting gas hits the surface of the NS. A reduction in the mass accretion rate relative to the Bondi rate is essential. Such a reduction has been seen in recent MHD simulations of spherically symmetric accretion with weak, dynamically unimportant magnetic fields (Igumenshchev \& Narayan 2002). The radial structure of the accretion flow is dramatically modified from the Bondi profile, and the mass accretion rate is decreased significantly. Other work on rotating flows also gives similar results ( $§ 2.3$ below).

Backed by the above observational and theoretical results, we perform a new calculation of the count rates for isolated NSs that accounts for the recent estimate of the velocity distribution, more realistic atmospheric spectra, as well as the correction to the Bondi rates predicted by the numerical simulations. We find that the resulting numbers are consistent with null detection at the ROSAT sensitivity.

\section{Dynamical evolution, emission properties and accretion rates}

The computation of the number counts requires several ingredients, and we describe each of them in detail in the following.

\subsection{Dynamical evolution and local space density of INSs}

As discussed in $\S 1$, in the last few years several groups (Lorimer, Bailes \& Harrison 1997; Hansen \& Phinney 1997; Cordes \& Chernoff 1998; Arzoumanian et al. 2002) have realized that the velocity distribution for pulsar velocities derived by Narayan \& Ostriker 
(1990; NO in the following) overpredicts the fraction of slow moving stars and underpredicts the contribution from faster moving ones. Here, we adopt the estimates by Cordes \& Chernoff (1998, CC in the following). Using a sample of 49 young pulsars, they found that the distribution $p\left(v_{R i}, v_{\phi i}, v_{z i}\right)$ for the velocities of their progenitors can be best described by a two-component gaussian model with characteristic speeds of $\sim 175 \mathrm{~km} / \mathrm{s}$ and $\sim 700 \mathrm{~km} / \mathrm{s}$, representing $\sim 86 \%$ and $14 \%$ of the population, respectively. The scale height for both components (assuming a gaussian distribution) is $\sim 0.13 \mathrm{kpc}$. The radial birth distribution is assumed to follow that of the stellar disk, that is an exponential with scale length $4.5 \mathrm{kpc}$ out to a maximum radius of $20 \mathrm{kpc}$.

For a given initial phase space distribution $f_{i}\left(R_{i}, z_{i}, v_{R i}, v_{\phi i}, v_{z i}\right)$, the equilibrium distribution can be determined by Monte Carlo simulations of the orbits of the stars in the galactic potential (e.g. Paczynski 1990; Blaes \& Rajagopal 1991). However, this is a very time-consuming method and, as BM demonstrated, an excellent approximation to the numerical simulations is provided by the use of Liouville's theorem under the "thin-disk approximation", where the motions along $R$ and $z$ can be decoupled. In this case, the equilibrium phase space distribution is related to the initial one through the relation

$$
\int d R d z d v_{R} d v_{\phi} d v_{z} f_{\mathrm{eq}}\left(L_{z}, E_{R}, E_{z}\right)=\int d R_{i} d z_{i} d v_{R i} d v_{\phi i} d v_{z i} f_{i}\left(R_{i}, z_{i}, v_{R i}, v_{\phi i}, v_{z i}\right)
$$

where the integrals are taken over the regions of phase space around given values of $L_{z}, E_{R}$, and $E_{z}$.

Figure 1 shows a comparison between the equilibrium speed distribution derived from the NO initial distribution, and the one derived from the $\mathrm{CC}$ birth distribution. It can be seen that there is a significantly larger fraction of high velocity stars. When using the birth parameters derived by CC, the local NS density $n_{\mathrm{NS}}(R=8.5 \mathrm{kpc}, z=0) \equiv n_{\mathrm{NS}}^{0}$ is reduced from the value $n_{\mathrm{NS}}^{0} \approx 7 \times 10^{-13} N_{\text {tot }} \mathrm{pc}^{-3}$ found by BM for the NO population, to $n_{\mathrm{NS}}^{0} \approx 4 \times 10^{-13} N_{\text {tot }} \mathrm{pc}^{-3}$.

It should be noted that the relation in equation (1) does not account for the fraction of high velocity stars that escape the Galaxy. As a matter of fact, this has very little effect on the statistics of the high- $\dot{M}$ tail of the accretion rate distribution, which is heavily dominated by the low- $v$ tail of the velocity distribution. What could be affected more significantly is the local density $n_{\mathrm{NS}}^{0}$ of stars, although this too is mostly determined by the slowest local stars. In fact, since most NSs are born in the vicinity of the Galactic plane and move away from it, the slowest stars, having smaller orbits, will pass more frequently through the detectable volume. A proper computation of the local density corrected for the fraction of escaped stars, would require a knowledge of the birth rate distribution during the lifetime of the Galaxy (as the oldest stars are more likely to have escaped than the 
younger ones). However, it is possible to place an upper limit to the error made in the local number density by neglecting the fraction of escaped NSs. This can be done by assuming that all NSs were born at $t=0$ (Galaxy birth time), and therefore all the ones with velocity larger than the escape velocity have abandoned the Galaxy by the present time. Using again equation (1) with a cutoff in the initial velocity distribution for $v>v_{\text {esc }}$, and for an escape velocity $\sim 500 \mathrm{~km} / \mathrm{s}$ (Cordes \& Chernoff 1998), we find that the correction to the local number density is within $10 \%$. This upper limit is well within the uncertainties due to other effects, and in particular the details of the low- $v$ tail of the velocity distribution which, according to the estimates made by other groups (e.g. Arzoumanian et al. 2002) can be more substantial ${ }^{6}$.

For the total number $N_{\text {tot }}$ of neutron stars populating the Galaxy at the present time, we use the estimate $N_{\text {tot }} \sim 10^{9}$ based on nucleosynthesis constraints (Arnett et al. 1989), and in particular on the condition that the number of Type II SNe in the past be sufficient to account for the total mass fraction of Oxygen observed in the Galactic disk today. Note that this implies that either the Type II SN rate was much higher in the past than it is today $^{7}$, or that the observed rate today is only a small fraction of the actual core collapse events $^{8}$.

\subsection{Emission properties of accreting NSs}

Previous investigations of the expected source counts from accreting isolated NSs have assumed a blackbody spectrum, although it has been recognized, since the early work of Zeldovich \& Shakura (1969), that the spectrum of accreting NSs will depart from a purely Planckian shape. Zampieri et al. (1995) worked out the case of pure $\mathrm{H}$ atmospheres with negligible fields at low luminosities, and found that the emerging spectrum is harder than a blackbody at $T_{\text {eff }}$, and the hardening increases as the temperature decreases. The spectral shape, as well as this behaviour with $T_{\text {eff }}$, is very similar to that found in unmagnetized, $\mathrm{H}$ atmospheres around cooling NSs (e.g. Romani 1987; Zavlin et al. 1996), as the hardening is not related to the heat source but to the frequency dependence of the free-free opacity. The

\footnotetext{
${ }^{6}$ The Arzoumanian et al. distribution has also a more substantial high velocity tail, and they find that about $50 \%$ of all pulsars will escape the Galaxy.

${ }^{7}$ The currently observed Type II supernova rate from external Sb and Sc galaxies is about one every 50 years (e.g. Vandenberg, McClure \& Evans 1987).

${ }^{8}$ Arnett et al. argue that this could be the case if many core collapse events were no more luminous than SN 1987A, or if a large fraction of them occurs in dense, obscuring regions, as in the case of Cas A.
} 
detailed spectra resulting from accretion at low rates onto a strongly magnetized NS have been computed by Zane et al. (2000). They found that the hard tail present in nonmagnetic models with comparable luminosity is suppressed, and the $\mathrm{X}$ ray spectrum is much closer to a blackbody.

For a given accretion luminosity, the geometry of the incoming flow is strongly dependent on the strength of the magnetic field of the star. In the simplest model (Davidson \& Ostriker 1973), one can assume that the radius of the accretion column is delineated by the field lines that pass through the Alfven radius. This leads to an area for the accretion region $\sim 10^{8} B_{12}^{-4 / 7} \dot{M}_{10}^{2 / 7} \mathrm{~cm}^{2}$, although it does not account for the fact that the Rayleigh-Taylor instability can lead to penetration of the accretion material through the field lines, resulting in much larger emitting areas (Arons \& Lea 1980). For definiteness, in our calculations we assume a polar cap of area $\sim 1 \mathrm{~km}^{2}$.

Given the considerations above, for the purpose of our analysis we use spectra of unmagnetized, hydrogen atmospheres when considering a model in which the emission occurs isotropically over the whole star (as would be typical of low magnetic fields), and blackbody spectra in a model where accretion occurs through the polar caps (at high magnetic fields). Note that, if accretion occurs through a fraction $f$ of the area of the star, then the corresponding temperature at the star surface will be larger by a factor $f^{-1 / 4}$. For completeness, we also consider another spectral model which is a combination of isotropic emission and a power-law. This is empirically motivated, as many accreting systems display evidence for both a power law and a thermal component in their spectra (White, Stella \& Parmar 1988), although note that the formation of standing shocks during the process of accretion can also give rise to significant power law tails (Zeldovich \& Shakura 1969). For definiteness and in agreement with observations (Campana \& Stella 2000), when considering this model, we assume that about half of the accretion energy gets reprocessed into a thermal component, and half comes out in a non-thermal, powerlaw component. For the latter, we adopt a photon index of 1.5, up to an energy of $\sim 10 \mathrm{keV}$.

\subsection{Accretion rates}

The standard Bondi formula for the accretion rate onto the surface of a compact star of mass $M$ is given by the expression

$$
\dot{M}_{\text {Bondi }} \approx \frac{4 \pi G^{2} M^{2} \rho}{\left(v^{2}+c_{s}^{2}\right)^{3 / 2}},
$$

where $c_{s}$ and $\rho$ are the sound speed and density of the ambient ISM, and $v$ is the velocity of the star with respect to the medium. Previous investigations of the statistics of accreting 
NSs have been based on the Bondi formula to compute accretion rates ${ }^{9}$. However, as discussed in $\S 1$, a measurement of the gas density in the vicinity of the black hole in Sgr $\mathrm{A}^{*}$ indicates that the Bondi expression highly overestimates the true accretion rate in that object.

The observational result on $\mathrm{Sgr} \mathrm{A}^{*}$ is supported by three-dimensional MHD simulations by Igumenshchev \& Narayan (2002) and Proga \& Begelman (2003) which show that, in the presence of a weak, dynamically unimportant, large-scale magnetic field in the external medium ${ }^{10}$, the structure of a spherically-symmetric radiatively inefficient accretion flow is dramatically different from the Bondi prediction. As the gas flows in, the magnetic field is amplified. When the field reaches equipartition, it begins to reconnect, heating the gas, and setting up turbulent motions. These motions, plus the tension in the amplified field, strongly suppress the accretion rate onto the black hole. The resulting accretion rate scales roughly as

$$
\dot{M} \sim\left(\frac{R_{\text {in }}}{R_{a}}\right)^{p} \dot{M}_{\text {Bondi }}
$$

where $R_{a} \sim G M / c_{s}^{2}$ is the "accretion radius" at which the ambient material begins to feel the gravitational influence of the accreting star, $R_{\text {in }}$ is an effective inner radius which is of order a few to few tens of Schwarzschild radii, and the index $p$ is uncertain but expected to be less than or of order unity.

The work described above refers to the infall of non-rotating or slowly-rotating magnetized gas, and is most relevant for the problem at hand. However, the main result from the simulations, namely that the accretion rate is strongly suppressed, is more general and has been noted also in many studies of strongly rotating flows. The evidence has come from a variety of hydrodynamic simulations - Stone, Pringle \& Begelman (1999; two-dimensional simulations with viscosity), Igumenshchev \& Abramowicz (2000; 2D simulations with $\alpha$ viscosity), Igumenshchev et al. (2000, 3D $\alpha$ viscosity), Proga \& Begelman (2002; 2D inviscid) — and MHD simulations — Stone \& Pringle (2001; 2D simulations), Machida, Matsumoto \& Mineshige (2001; 2D and 3D), Hawley, Balbus \& Stone (2001; 3D), Igumenshchev, Narayan \& Abramowicz (2003; 3D). All of these studies indicate a reduced $\dot{M}$, as in equation $(3)$, with $p$ in the range $0.5-1$.

\footnotetext{
${ }^{9}$ See however Rutledge (2001) for a discussion of a subpopulation of highly magnetic $\left(B \lesssim 10^{14} G\right)$, accreting NSs whose accretion rate scales as $v^{1 / 3}$ rater than $v^{-3}$ as in Bondi.

${ }^{10}$ In their simulations, Igumenshchev \& Narayan assumed that the initial interstellar field had magnetic pressure on the order of 1 percent of the gas pressure. If the field is stronger, then the effects are only more dramatic.
} 
Two different explanations have been advanced for the reduction in $\dot{M}$ in radiatively inefficient accretion flows: (i) winds and outflows (Blandford \& Begelman 1999; Narayan \& Yi 1994, 1995), (ii) convection (Narayan et al. 2000; Quataert \& Gruzinov 2000). In the former explanation, gas accretes at the Bondi rate at the accretion radius $R_{a}$. However, as the gas flows in towards smaller radii, much of it is lost to an outflow so that only a tiny fraction finally reaches the central star. In the latter explanation, convection sets up a barrier to accretion, such that the net accretion rate is reduced at all radii out to $R_{a}$. Note that, within the latter scenario, a stationary solution can be obtained as convection changes the conditions at $R_{a}$ by increasing the pressure and preventing the gas from falling in supersonically. The physical picture is somewhat similar to that envisaged by Ostriker et al. (1976), where preheating of the infalling gas by the emergent X rays can suppress accretion (see in this regard also Blaes, Madau \& Warren 1995). Detailed MHD simulations provide support for both the outflow picture (Stone \& Pringle 2001; Hawley et al. 2001) and the convection idea (Machida et al. 20001; Igumenshchev et al. 2003). It is possible that both effects operate, e.g., with convection driving an outflow.

All the simulations described above refer to accretion onto a black hole. Most of the results are expected to carry over to the NS case. If the star is unmagnetized, the estimate of $\dot{M}$ given in equation (3) should be valid with essentially no change. If the star is strongly magnetized, then equation (3) will only be valid under the conditions for which the star is able to accrete at a steady pace. Indeed, in order for a rotating, magnetic star to be able to accrete steadily, three conditions need to be satisfied (BM, Popov et al. 2000, Treves et al. 2000, Ikhsanov 2003). Firstly, the Alfven radius $r_{A}$ (where the magnetic energy density is comparable to the kinetic energy of the accreting gas) must not exceed the accretion radius $R_{a}$, otherwise magnetic pressure would dominate everywhere over the gravitational pull, and the star would be in the so-called georotator state ${ }^{11}$ (Liuponov 1992). This condition is typically fulfilled for the stars in the low- $v$ tail of the velocity distribution, which dominate the accretion statistics. Once the constraint $r_{A}<R_{a}$ is satisfied, there are still two barriers that need to be overcome for accretion to proceed steadily, that is: (i) at the accretion radius, the ram pressure of the accreting material must be larger than the radiation pressure from the pulsar (otherwise the star would be in the ejector phase), and (ii) the velocity of the magnetosphere at the Alfven radius must be smaller than the Keplerian velocity at that same radius. If this latter condition were not satisfied, the star would act as a propeller and prevent material from penetrating inside $r_{A}$ (Illiaronov \& Sunyaev 1975).

The relative importance of the various phases discussed above during the life of the NS,

\footnotetext{
${ }^{11}$ This regime was also investigated by Toropina et al. (1991), who called it "magnetic plow". Some accretion may occur in this regime due to 3D MHD instabilities (Arons \& Lea 1976, 1980).
} 
depends on a combination of its velocity, spin period, magnetic field, and accretion rate. In particular, the strength and the evolution of the magnetic field in the star play an especially important role. Livio et al. (1998) and Colpi et al (1998) first pointed out that significant field decay can cause the NS to linger in the ejector or propeller state for a time longer than the lifetime of the Galaxy. The fraction of stars which can be in the accreting phase at the present time is therefore sensitive to both the initial value of the magnetic field, and to its decay time. For an initial field strength on the order of $10^{12} \mathrm{G}$, and an intermediate value of the decay time $\tau_{d} \sim 10^{9} \mathrm{yr}$, they found that about half of the stars are allowed to accrete at the present time. As a matter of fact, the question of whether the magnetic field of the stars decays is, by itself, a very controversial issue, both on theoretical and on observational grounds. Theoretical predictions range from an exponential/power-law decay of the field (e.g. Ostriker \& Gunn 1969; Goldreich \& Reisenegger 1992; Urpin \& Muslimov 1992) to little or no decay at all within the lifetime of the Galaxy (e.g. Romani 1990; Srinivasan et al. 1990). Observationally, low magnetic fields $\left(\sim 10^{9} \mathrm{G}\right.$ or so), possibly the result of a decay, have been inferred in low-mass X-ray binaries and millisecond pulsars, but no firm conclusions have been established yet for isolated objects. However, if evolution occurs but still leaves a residual field $B \sim 10^{8}-10^{9} \mathrm{G}$, a substantial fraction of the population should still be able to accrete at the present time (BM, Colpi et al. 1998). Keeping these uncertainties in mind, here we assume that all the NSs have reached the accretion phase at the present time and are accreting at the rate given by equation (3). As a matter of fact, it is sufficient that this condition holds only for a substantial fraction of the the low- $v$ tail of the velocity distribution, which is what dominates the accretion statistics.

For the calculations at hand, we need an estimate of the factor by which the mass accretion rate $\dot{M}$ onto the NS is suppressed relative to the Bondi rate. This requires an estimate of $R_{\mathrm{in}}$ and $p$ in equation (3). Abramowicz et al. (2002) found that $R_{\mathrm{in}}$ is of order 50 Schwarzschild radii for a rotating convection-dominated flow; $R_{\text {in }}$ is probably somewhat smaller, by a factor of a few, for a non-rotating flow. A more serious uncertainty is in the value of $p$. The outflow model does not make any specific prediction other than that $p$ should lie somewhere in the range 0 to 1 (Blandford \& Begelman 1999). The idealized convection-dominated accretion flow model, on the other hand, predicts a precise value: $p=1$ (Narayan et al. 2000; Quataert \& Gruzinov 2000). Numerical simulations give a range of values for $p$, between about 0.5 and 1 ; the deviation from unity may be explained within the convection model as the result of "vertical" rather than "radial" convection (Igumenshchev et al. 2003). Finally, we note that a recent analysis of Sgr A* (Yuan et al. 2003) indicates that an accretion model with $p \sim 0.3$ fits the observed spectral data and polarization constraints fairly well.

Given the uncertainties, especially in the value of $p$, it is not possible to derive a precise 
estimate of $\dot{M}$ for the accreting isolated NS problem. The only statement one can make with confidence, based on the evidence at hand, is that a radiatively inefficient accretion flow will not have $\dot{M}$ equal to the Bondi rate; the accretion rate is always suppressed, but it is not clear by precisely how much. We thus try a range of suppression factors in this paper: $\dot{M}=\left(10^{-2}, 10^{-3}, 10^{-4}\right) \dot{M}_{\text {Bondi }}$, with $10^{-3} \dot{M}_{\text {Bondi }}$ as our best-guess estimate. The selected range corresponds roughly to $p \sim 0.5-1$.

\section{Source counts: theory confronts observations}

To derive the probability distribution for the accretion rate, $f(\dot{M}, r)$, at distance $r$ from us, we also need an estimate for the distribution $n_{\mathrm{HI}}(r)$ of hydrogen in the ambient medium surrounding the solar region. Measurements of interstellar absorption (Paresce 1984; Warwick et al. 1993) suggest that the local interstellar medium consists of smoothly distributed gas with density $\sim 0.07 \mathrm{~cm}^{-3}$ up to a distance of about $100 \mathrm{pc}$, and denser gas, $\sim 0.5-1 \mathrm{~cm}^{-3}$ beyond that. As a representative line of sight to compute source counts per unit solid angle, we will consider a line of sight with $n_{\mathrm{HI}}(r)=0.07 \mathrm{~cm}^{-3}$ for $r \leq 100 \mathrm{pc}$ and

$n_{\mathrm{HI}}(r)=1 \mathrm{~cm}^{-3}$ for $r>100 \mathrm{pc}$. Whereas this is a good approximation as an average in the local bubble, as a matter of fact, the ISM is multiphase and comprised of cold (neutral) gas, as well as warm and hot (ionized) gas. The hotter component is more tenuous, and as such the accretion luminosity for stars embedded within it is further reduced. On the other hand, hot gas does not contribute much to photoelectric absorption, and the resulting larger sampling depth along ionized lines of sight tends to counterbalance the reduction in luminosity. Here we have assumed for simplicity all the material to be neutral. A detailed modelling of the various ISM phases, which would vary depending on the line of sight under consideration, is beyond the scope of this work.

Let now $C$ be the detector count rate. The number of sources along a given line of sight which are detectable above the threshold $C$ is

$$
\frac{d N}{d \Omega}(>C)=n_{\mathrm{NS}}^{0} \int_{0}^{\infty} d \dot{M} \int_{0}^{d(\dot{M}, C)} d r r^{2} f(\dot{M}, r)
$$

where the maximum distance $d(\dot{M}, C)$, up to which sources above the threshold $C$ can be detected, is obtained by solving the implicit equation

$$
C=\frac{1}{4 \pi d^{2}(\dot{M}, C)} \int_{0}^{\infty} L(\nu, \dot{M}) A_{\mathrm{eff}}(\nu) e^{-\tau(\nu)} \frac{d \nu}{h \nu} .
$$

Here $L(\nu, \dot{M})$ is the luminosity of the star, $A_{\text {eff }}(\nu)$ is the energy-dependent effective area of the detector under consideration, and $\tau(\nu)$ is the photoelectric absorption to the source, 
which we compute using the fit to the cross sections by Morrison \& McCammon (1983). For the ROSAT PSPC response, we have taken the version pspcb-gain1-256 ${ }^{12}$ and used it in Eq.(5) by performing an interpolation to the data.

As a first step towards estimating the contribution of the various effects described above to the source statistics, in Figure 2 we compare the number counts obtained under the same assumptions made by BM (i.e. NO pulsar velocity distribution, Bondi accretion rate, and blackbody spectra) with those obtained assuming the $\mathrm{CC}$ velocity distribution and atmospheric spectra but still with the Bondi accretion rate. As the figure shows, despite the reduction in the number counts due to the higher mean velocity, the predicted rates are still well above the limits set by the ROSAT All Sky Survey. In particular, in the case of isotropic emission with atmospheric spectra, the reduction in the counts due to the higher mean velocities is considerably offset by the increase due to the atmospheric rather than BB spectra. In fact, as explained above, for the same value of the star temperature, the atmospheric spectra are harder than BB, hence producing more emission in the X-ray band.

In Figure 3, we show the predicted source counts once the correction to the Bondi rate discussed in $\S 2.3$ is introduced. Note that, while in Figure 2 we showed the counts per unit solid angle, here we have considered the numbers over the entire sky. This is because, in this case, the distances over which the sources are detectable are comparable to the size of the local bubble, over which the density distribution can be taken as isotropic, and therefore the line of sight that we have used can be considered as representative of the whole sky. It can be seen from Figure 3, that the predicted counts are consistent with a null detection at the ROSAT PSPC sensitivity.

As discussed in $§ 2.3$, a rough estimate for the reduction in the Bondi rate is $\sim 10^{-3}$; however, for completeness, we explore the effects on the source counts for the range of $\dot{M} \sim 10^{-2}-10^{-4} \dot{M}_{\text {Bondi }}$. This is shown in Figure 4, for the case of polar cap emission and the CC velocity distribution. Whereas for $\dot{M} \lesssim 10^{-3} \dot{M}_{\text {Bondi }}$ the counts are consistent with no detection at the ROSAT sensitivity, a rate $\dot{M} \sim 10^{-2} \dot{M}_{\text {Bondi }}$ would still require that a good fraction of the sources is not in the accretion phase for most of the time.

\footnotetext{
${ }^{12} \mathrm{ftp}: / /$ legacy.gsfc.nasa.gov/caldb/data/rosat/pspc/cpf/matrices/
} 


\section{Summary}

We have revisited the problem of the statistics of old and isolated neutron stars, which ought to be detectable in X-rays as a result of accretion from the ISM. We have used the recent estimates for the velocity distribution made by Cordes \& Chernoff (1998), we have gone beyond the blackbody assumption for the spectra, using atmospheric models, and we have used a "revised Bondi" accretion rate, according to the results of MHD simulations and supported by direct observations in the case of accretion around supermassive black holes. Whereas the first two effects together do not result in a significant reduction of the predicted number counts, the modification to the Bondi formula dramatically reduces the number of observable sources.

As discussed in $§ 2.3$, other effects can also influence the detection statistics. Particularly important is the role played by the magnetic field of the star. This determines the spin evolution of the star itself, and the consequent fraction of the lifetime during which the star can be in a regime of steady accretion, rather than in other phases such as ejector or propeller. The importance of these various phases during the evolution of the NS life strongly depends on the evolution of the magnetic field within the star, which is by itself a very controversial issue. The source counts that we have computed here should therefore be considered as upper limits in that they are representative of a population that is accreting for most of its lifetime.

The number of observable sources that we have derived, with the inclusion of the revised velocity distribution, atmospheric spectra and reduced Bondi rate, is consistent with the detection of only a few candidates in the ROSAT all sky survey. As of now, there are only two objects which have been confirmed to be isolated NSs (see Rutledge et al. (2003) for a comprehensive review of the observations so far); moreover it is hard to firmly establish that they are actually powered by accretion and not by cooling. In the X-ray range, the spectrum from an accreting star looks quite similar to that of a cooling NS; however, Zane et al. (2000) noted that the two scenarios can be distinguished from optical observations, as accretion spectra have been found to display an excess emission over the best fitting blackbody X-ray distribution, which, on the other hand, does not appear in cooling models with similar temperatures.

We thank Omer Blaes, Bob Rutledge, Roberto Turolla and Silvia Zane for very useful discussions related to various aspects of this work. We particularly thank Omer Blaes for providing us with a routine for the space density distribution. We also thank the referee for helpful comments on the manuscript. RP aknowledges support by the Harvard Society of Fellows and a research grant by the Harvard-Smithsonian Center for Astrophysics. RN was 
supported in part by NASA grant NAG5-10780.

\section{REFERENCES}

Abramowicz, M. A., Igumenshchev, I. V., Quataert, E. \& Narayan, R. 2002, ApJ, 565, 1101

Arnett, W. D., Schramm, D. N. \& Truran, J. W. 1989, ApJ, 311, 258

Arons, J., \& Lea, S. M. 1976, ApJ, 207, 914

Arons, J., \& Lea, S. M. 1980, ApJ, 235, 1016

Arzoumanian, Z, Cordes, J. M. \& Chernoff, D. F. 2002, ApJ, 568, 289

Blaes, O. \& Rajagopal, M. 1991, ApJ, 381, 210

Blaes, O. \& Madau, P. 1993, ApJ, 403, 690

Blaes, O., Madau, P. \& Warren, O. 1995, ApJ, 454, 370

Blandford \& Begelman 1999, MNRAS, 303L, 1

Bower, G. C., Wright, M. C. H., Falcke, H., \& Backer, D. C. 2003, ApJ, in press (astro-ph/0302227)

Campana, S. \& Stella, L. 2000, ApJ, 541, 849

Colpi M., Turolla R., Zane S., Treves A. 1998, 1998, ApJ, 501, 252

Cordes, J. M. \& Chernoff, D. F. 1998, ApJ, 505, 315

Davidson, K., \& Ostriker, J. P. 1973, ApJ, 179, 585

Di Matteo, T., Carilli, C. L., \& Fabian, A. C. 2001, ApJ, 547, 731

Di Matteo, T., Quataert, E., Allen, S. W., Narayan, R., \& Fabian, A. C. 2000, MNRAS, 311, 506

Fabian, A. C., \& Canizares, C. R. 1988, Nature, 333, 829

Fabian, A. C., \& Rees, M. J. 1995, MNRAS, 277, L55

Goldreich, P. \& Reisenegger, A. 1992, ApJ, 395, 250

Hansen, B. M. S. \& Phinney, E. S. 1997, MNRAS, 291, 569

Hawley, J. F., Balbus, S. A., \& Stone, J. M. 2001, ApJ, 554, L49

Ichimaru, S. 1977, ApJ, 214, 840

Illarionov, A. \& Sunyaev, R., 1975, A\&A, 39, 185

Igumenshchev, I. V., \& Abramowicz, M. A. 2000, APJS, 130, 463 
Igumenshchev, I. V., Abramowicz, M. A., \& Narayan, R. 2000, ApJ, 537, L27

Igumenchev, I. V. \& Narayan, R. 2002, ApJ, 566, 137

Igumenshchev, I. V., Narayan, R., \& Abramowicz, M. A. 2003, ApJ, submitted (astro-ph/0301402)

Ikhsanov, N. R., 2003, A\&A in press, astro-ph/0301076

Liuponov, 1992, in "Astrophysics of Neturon Stars" (New York: Springer)

Livio M., Xu C. Frank J., 1998, ApJ, 492, 298

Loewenstein, M., Mushotzky, R. F., Angelini, L., Arnaud, K. A., \& Quataert, E. 2001, ApJ, $555, \mathrm{~L} 21$

Lorimer, D. R., Bailes, M., \& Harrison, P. A. 1997, MNRAS, 289, 592

Madau, P. \& Blaes O. 1994, ApJ, 423, 748

Machida, M., Matsumoto, R., \& Mineshige, S. 2001, PASJ, 53, L1

Narayan, R. 2002, in Lighthouses of the Universe, eds. M. Gilfanov, R. Sunyaev, E. Churazov, Springer, p405

Narayan, R. \& Ostriker, J. P. 1990, ApJ, 352, 222

Narayan, R., \& Yi 1994, ApJ, 428, L13

Narayan, R., \& Yi 1995, ApJ, 444, 231

Narayan, R., Yi, I., \& Mahadevan, R. 1995, Nature, 374, 623

Narayan, R., Igumenshchev, I. V., \& Abramowicz, M. A. 2000, ApJ, 539, 798

Ostriker, J. P. \& Gunn, J. E. 1969, ApJ, 157, 1395

Ostriker, J.P., Weaver, R., Yahil, A., McCray, R. 1976, ApJ, 208L, 61

Ostriker, J. P., Rees, M. J., \& Silk, J. 1970, Astrophys. Lett., 6, 179

Paczynski, B. 1990, ApJ, 348, 485

Paresce, F. 1984, AJ, 89, 1022

Popov, S.B., Colpi, M., Treves, A., Lipunov, V. M., Prokhrov, M .E. 2000, ApJ, 530, 896

Proga, D., \& Begelman, M. C. 2002, ApJ, 582, 69

Proga, D., \& Begelman, M. C. 2003, submitted to ApJ, preprint astro-ph/0303093

Quataert, E. \& Gruzinov, A. 2000, 539, 809

Rees, M. J., Begelam, M, C., Blandford, R. D. \& Phinney, E. S. 1982, Nature, 295, 17

Romani, R. W. 1987, ApJ, 313, 718 
Rutledge, R. E. 2001, ApJ, 553, 796

Romani, R. W. 1990, Nature, 347, 741

Rutledge, R. E., Fox, D. W., Bogosavljevic, M., Mahabal, A. 2003, astro-ph/0302107

Srinivasan, G., Bhattacharya, D., Muslimov, A. G., \& Tsygan, A. I. 1990, Curr. Sci, 59, 31

Stone, J. M., \& Pringle, J. E. 2001, MNRAS, 322, 461

Stone, J. M., Pringle, J. E., \& Begelman, M. C. 1999, MNRAS, 310, 1002

Toropina, O. D., Romanova, M. M., Toropin, Yu. M., Lovelace, R.V. J. 2001, ApJ, 501, 964

Treves, A. \& Colpi, M. 1991, A\&A, 241, 307

Treves, A., Turolla, R., Zane, S., Colpi, M. 2000, PASP, 112, 297

Urpin, V. A. \& Muslimov, A. G. 1992, MNRAS, 256, 261

Vandenberg, S., McClure, R., \& Evans, R. 1987, ApJ, 323, 44

Warwick, R. S., Barber, C. R., Hodgkin, S. T., \& Pye, J. P. 1993, MNRAS, 262, 289

White, N. E., Stella, L. \& Parmar, A. N. 1988, ApJ, 324, 363

Yuan, F., Quataert, E., \& Narayan, R. 2003, ApJ, submitted (astro-ph/0304125)

Zampieri, L, Turolla, R., Zane, S., \& Treves, A. 1995, ApJ, 439, 849

Zane, S., Turolla, R., Zampieri, L., Colpi, N., Treves, A. 1995, ApJ, 451, 739

Zane, S., Turolla, R. \& Treves, A. 2000, ApJ, 537, 395

Zavlin, V. E., Pavlov, G. G., Shibanov, Y. A. 1996, A\&A, 315, 141

Zeldovich, Y., \& Shakura, N.1969, Soviet Astron.-AJ, 13, 175 


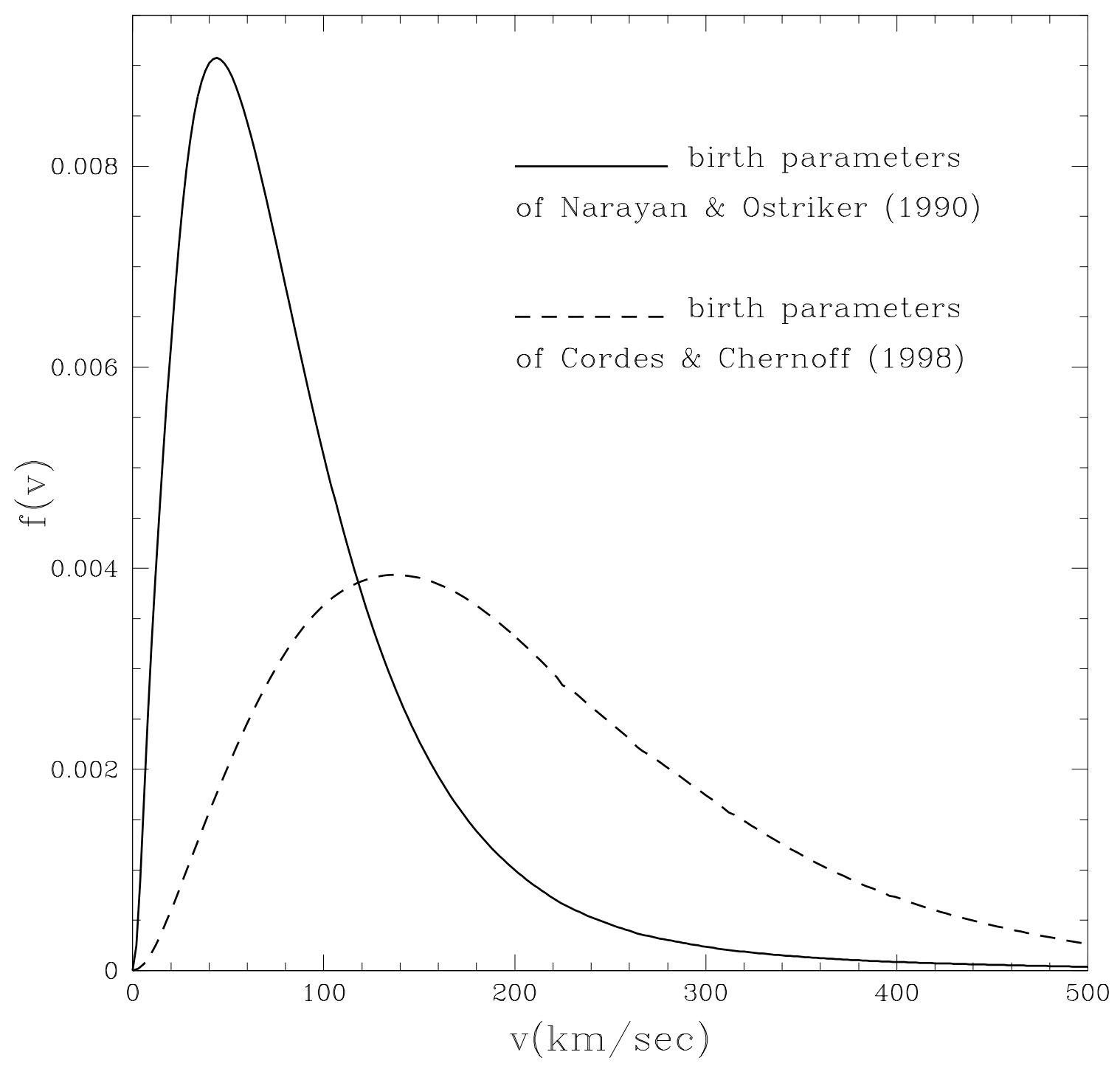

Fig. 1.- Comparison between the speed distribution of NSs in the solar neighborhood $(R=8.5 \mathrm{kpc}, z=0)$ obtained assuming the Narayan \& Ostriker (1990) initial distribution and the Cordes \& Chernoff (1998) distribution. 


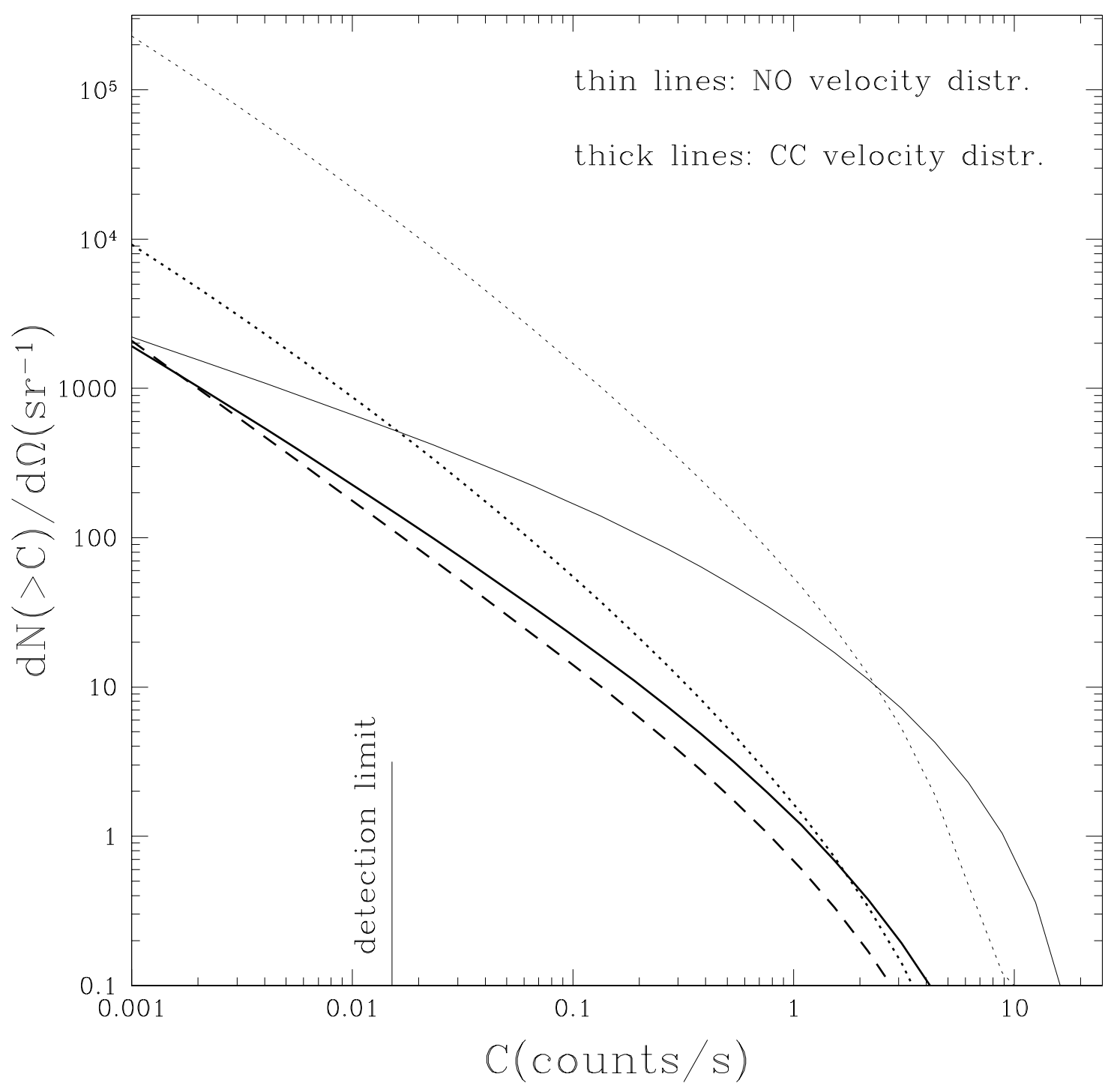

Fig. 2.- The combined effect on source statistics of the higher mean velocities and various spectral energy distributions, shown for the cumulative counts in the 0.1-2.4 keV band of the ROSAT PSPC detector. The thin lines assume the NO velocity distribution and BB spectra both for the case of isotropic emission (solid line) and polar cap emission (dotted line). This is the case studied by BM. The thick lines assume the $\mathrm{CC}$ velocity distribution and consider various possiblities for the spectral energy distribution: blackbody in the case of polar cap emission (dotted line), unmagnetized hydrogen atmosphere in the case of isotropic emission (solid line), and a combination of isotropic, atmospheric emission with a power law (dashed line). In all cases, the accretion rate is assumed to be equal to the Bondi rate. In the case of isotropic emission, for count rates $\lesssim 0.001 \mathrm{~s}^{-1}$, the reduction in the counts due to the increased mean velocity is compensated by the increase due to the harder atmospheric spectrum. 


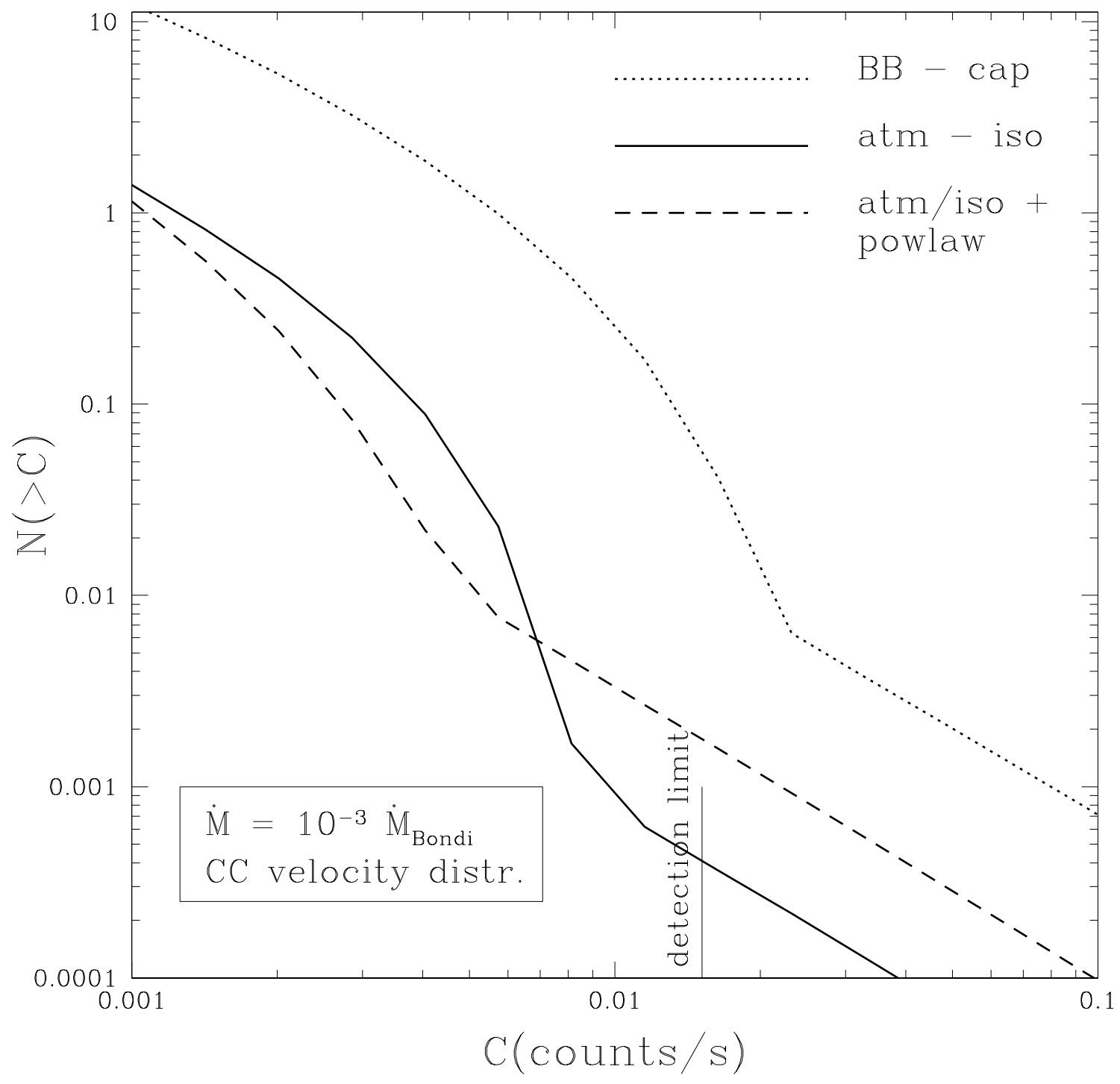

Fig. 3.- Cumulative source counts in the $0.1-2.4 \mathrm{keV}$ band of the ROSAT PSPC detector over the entire sky (assuming that the line of sight that we have considered is representative of the whole sky over a small distance scale). The computation of these counts includes the CC velocity distribution, an atmospheric spectrum for the isotropic component of the emission, and a mass accretion rate reduced below the Bondi rate by a factor of $10^{-3}$, according to the discussion in $§ 2.3$. 


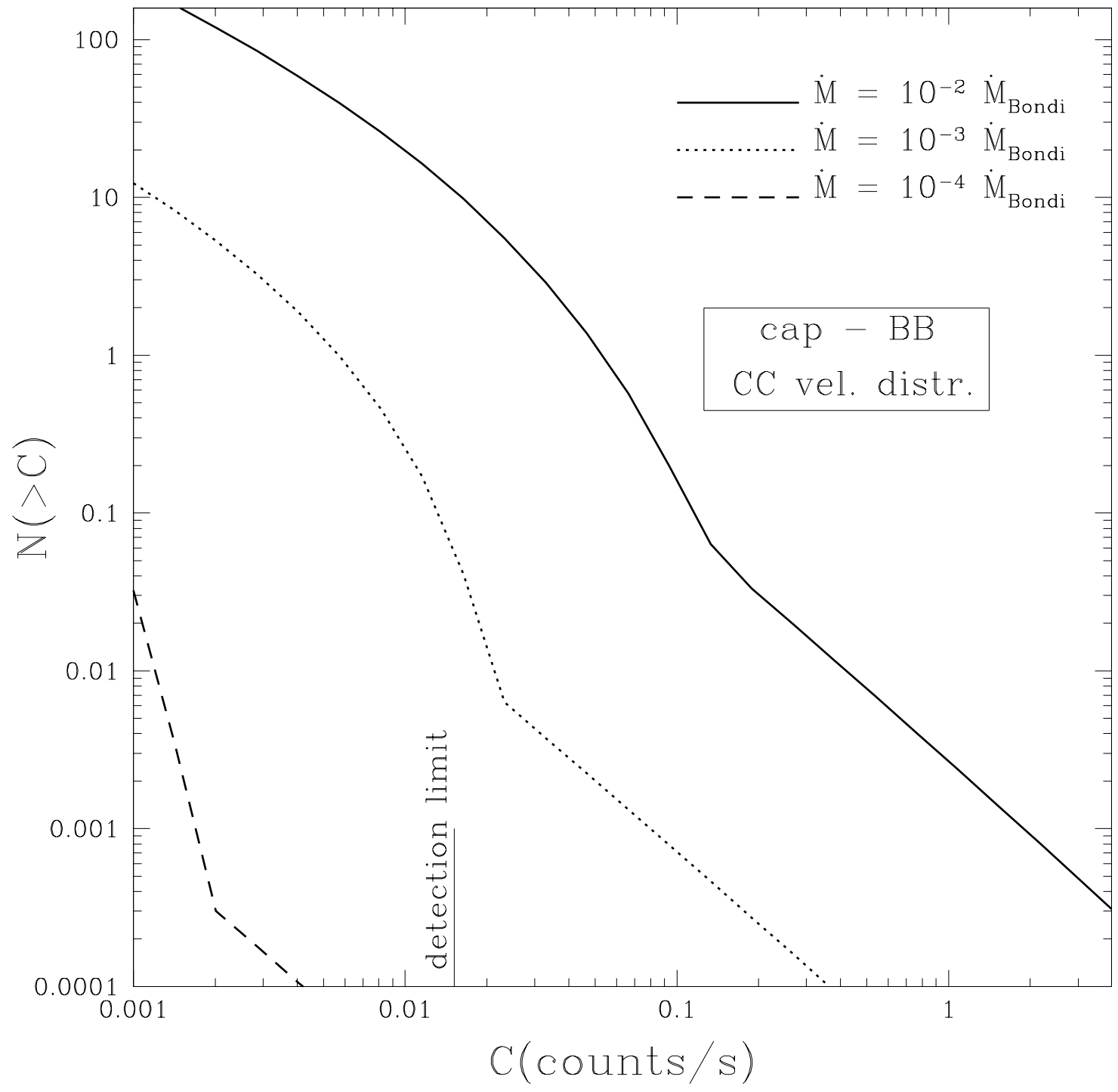

Fig. 4. - The effect of a suppressed mass accretion rate on the number counts in the 0.1-2.4 $\mathrm{keV}$ band of the ROSAT PSPC detector, shown for the case of polar cap emission with a blackbody spectral energy distribution, and for the $\mathrm{CC}$ velocity distribution. 